\title{
A Dielectric Resonator Antenna Array for Wideband Applications
}

\author{
Sagar Erande ${ }^{1}$, Syed Amir $\mathrm{Ali}^{2}$ \\ ${ }^{1,2}$ (Amity School of Engineering and Technology, Amity University Uttar Pradesh, India)
}

\begin{abstract}
This paper presents a new approach to design the dielectric resonator antenna (DRA) for wideband applications, which has a stepped construction the hexagon shaped upper resonator and triangle shaped lower resonator. The DRA is excited by a conformal inverted-trapezoidal patch connected to a microstrip line. The wider bandwidth is achieved, because of three factors: compact honeycomb and triangle shaped dielectric resonator, a conformal inverted-trapezoid patch as a feed mechanism, and a copper-clad substrate as a baseboard. A $76.25 \%$ matching bandwidth $\left(S_{11}<10 \mathrm{~dB}\right)$ is achieved with broadside radiation patterns where the impedance bandwidth covers a frequency range from 5.1 to $11.1 \mathrm{GHz}$. Based on the proposed structure, a fourelement array is designed, and the degree of the directivity increases largely.
\end{abstract}

Keywords: dielectric resonator antenna, hexagonal upper resonator, triangle shaped lower resonator, inverted trapezoidal patch, DRA

\section{INTRODUCTION}

A resonant antenna was proposed by Professor S. A. Long in 1983. The dielectric resonator antennas (DRAs) offer several advantages, such as small size, low dissipation loss. Since the DRA has negligible metallic loss, it is highly efficient when operated at millimeter wave frequencies. The shapes of DRAs are various-simple ones, such as a hemispherical DRA [1], a cylindrical DRA [2] and a rectangular DRA [3], and improved ones, such as truncated tetrahedron shape, split cone shape and half-hemispherical shape DRAs. DRAs can be excited with different feeding mechanism, such as coaxial feed, aperture, microstrip line and coplanar lines [4]. As the present and future communication systems have imposed strict requirements on the bandwidth of antennas, a lot of bandwidth enhancement techniques are used in designing the dielectric resonator antenna (DRA), mainly at lowering the inherent Q-factor of the resonator; using external matching networks; and combining multiple resonators. A much wider bandwidth was achieved by stacking two different DRAs [5, 6], loading a high permittivity, low-profile dielectric disc on top of a conventional homogeneous DRA in and plugging an inner core into the lower stacked part [7]. In addition, multi-segment DRAs are developed to enhance its coupling to a microstrip line by inserting one or more thin segments of different permittivity substrates under a DRA of low permittivity [8].

In this paper, we present a new design in which the homogenous resonator structure is consisted of two parts: the upper part has a hexagon -liked shape and the lower part has a triangle shape and the feeding mechanism is a conformal inverted-trapezoidal patch connected to a microstrip line with an intermediate substrate (low permittivity) inserting technique. The DRA has an obvious advantage of small size, and the simulated results demonstrate that the proposed DRA achieves a wider impedance bandwidth as much as $78 \%$ with a broadside radiation pattern. In order to increase the degree of directivity, the array composed of 14 elements are designed.

In Section 2, antenna geometry is introduced clearly and the simulated results are given in detail. In Section 3, an array is designed by using the proposed novel DRA elements, and the characteristics of DRA array will be described. In the last section, conclusions are concluded.

\section{ANTENNA CONFIGURATION}

Fig. 1 shows the schematic of the proposed DRA and Fig. 2 shows the top view, where a stepped dielectric resonator is centrally placed on a square substrate with a size of $30 \mathrm{~mm} \times 30 \mathrm{~mm}$.

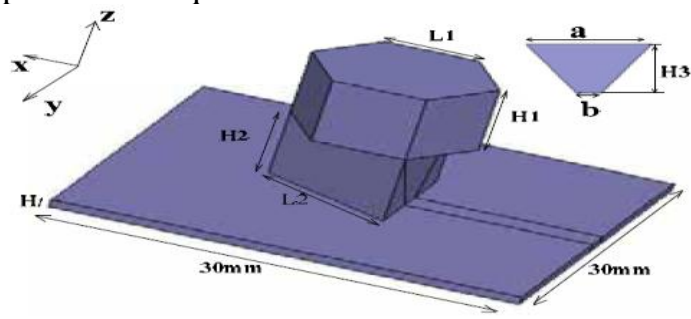

Figure 1: Schematic of DRA 


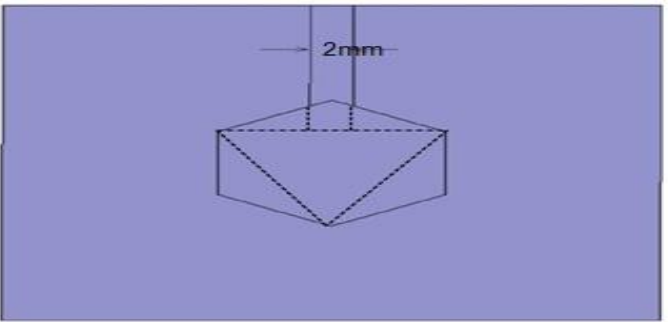

Figure 2: Top View of DRA

with respect to the central frequency at $7 \mathrm{GHz}$. The upper part of DR is a hexagon -shaped DR with equal six-side length $\mathrm{L} 1=6 \mathrm{~mm}$, and height $\mathrm{H} 1=6.35 \mathrm{~mm}$. The lower part is an equiangular-triangle across section with the height $\mathrm{H} 2=6.5 \mathrm{~mm}$, and length $\mathrm{L} 2=10.4 \mathrm{~mm}$. The DR is fabricated on Arlon $600(\mathrm{tm})$ microwave dielectric material with a permittivity of $\varepsilon_{r}=6$. The excitation mechanism adopts a conformal inverted-trapezoid patch attached on one side of the lower layer DR in the x-axis direction and connected to a 50 microstrip feed line. The impedance bandwidth can be enhanced by using a single-sided copper-clad substrate corresponding to the ground plane .

Using a single-sided copper-clad substrate means inserting a dielectric material between the DR and the ground plane, where the inserted substrate dielectric constant is much lower than the DR one that enables correct excitation of the fundamental mode. An appropriate wideband design of the proposed DRA adopts two methods. The numerical analysis was performed using the commercial full-wave analysis tool Ansoft HFSS employing the finite element method (FEM). Fig. 3 shows the return loss $\left(s_{11}\right)$ of the depicted DRA.

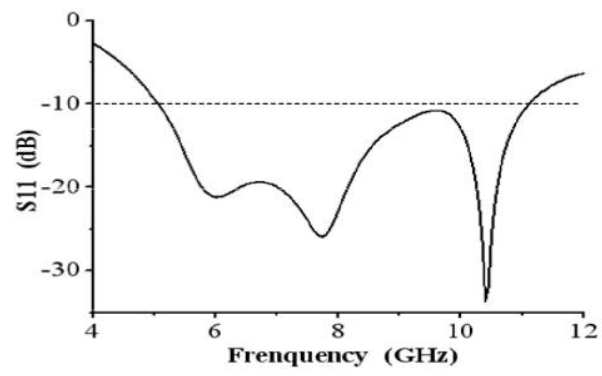

Figure 3. Return loss $\left(S_{11}\right)$ of DRA

The matching frequency range of the simulated homogeneous DRA is from $5.1 \mathrm{GHz}$ to $11.1 \mathrm{GHz}$ $\left(\mathrm{S}_{11}<10\right)$, corresponding to a bandwidth of $76.25 \%$.

Fig. 4 shows the radiation pattern at a resonance frequency of $6 \mathrm{Ghz}$. It can be seen that the radiation pattern is symmetrical in the $y-z$ plane (pi=90deg), and in the $\mathrm{x}-\mathrm{z}$ plane (pi=0deg) mainly due to the asymmetry of the structure in the $\mathrm{x}-\mathrm{z}$ plane.

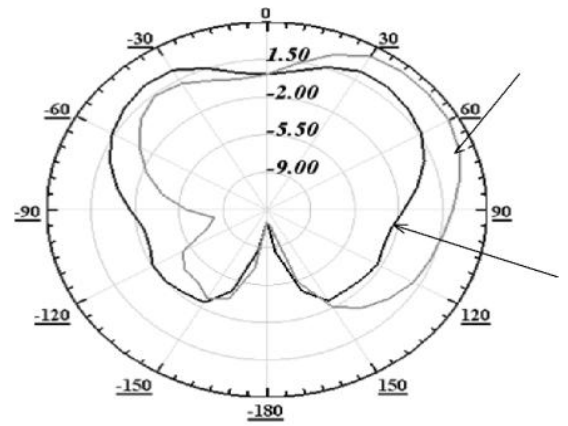

Figure 4: Simulated radiation pattern $(\mathrm{db})$

\section{DESIGN OF AN ARRAY}

To increase the degree of the directivity, an array is designed with four proposed DRAs. Fig. 5 shows the geometry of the array, and the microstrip corporate feed is used for feed arrangement. The first balanced line along a distance of quarter wavelength has a characteristic impedance of 70.7, and the second balanced line along a distance of 5/4 wavelength distance has a characteristic impedance of 50 . The formation of a directional DRA array requires that the DRA elements be positioned with a close spacing between elements. However, the proximity of the elements results in mutual coupling between elements due to the electromagnetic 
interaction between elements. The mutual coupling may be significant so that it substantially affects the performance of the array including radiation pattern, directivity, operating frequency and bandwidth. Hence, the spacing between elements is $0.7 \lambda_{0}$.

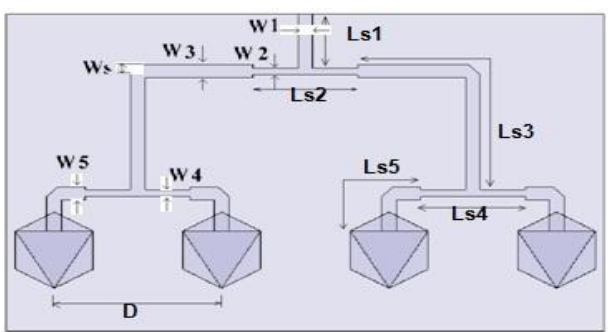

Figure 5. Geometry of the proposed antenna array

With the following DRA element and array parameters: DRA: $\mathrm{H} 1=6.35 \mathrm{~mm}, \mathrm{~L} 1=6 \mathrm{~mm}, \mathrm{H} 2=6.5 \mathrm{~mm}$, L2 $=10.4 \mathrm{~mm}$, Fig. 6 plots the simulated return loss $\left(\mathrm{S}_{11}\right)$.

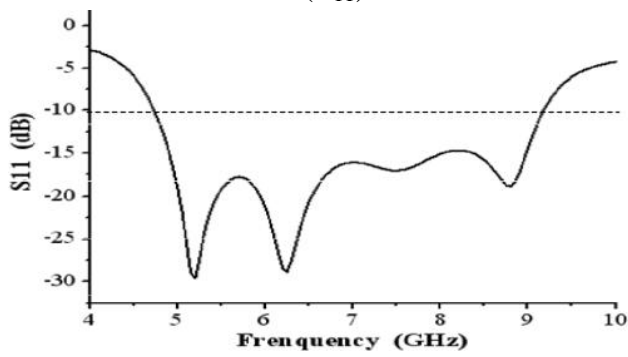

Figure 6. Simulated return loss (S11) of the array

\section{CONCLUSION}

A hexagonal DRA with advantages of compact structure which the area is $30 \mathrm{~mm}$ X $30 \mathrm{~mm}$ is presented and a larger bandwidth of $76.25 \%$ range from $5 \mathrm{GHz}$ to $11.1 \mathrm{GHz}$ is obtained. An inverted-trapezoidal patch excitation has a better impedance matching obviously. The array consisted of the proposed DRA has an increasing of the degree of the directivity and the impedance bandwidth curves is more smooth in the pass band, which can be used in wide band communication.

\section{REFERENCES}

[1] Kishk A.A., Zhou G., and Glisson A.W., "Analysis of dielectric-resonator antennas with emphasis on hemispherical structures," IEEE Antennas Propagation Magazine, 36(2), 1994, 20-31.

[2] Kishk A.A., "Wide-band truncated tetrahedron dielectric resonator antenna excited by a coaxial probe," IEEE Trans. Antennas Propagation, 51(10), 2003, 2913-2917.

[3] Kishk A.A., Yan Y., and Glisson A.W., "Conical dielectric resonator antennas for wide-band applications," IEEE Trans. Antennas Propagation, 2002, 50(4), 469-474.

[4] Junker G.P., Kishk A.A., and Glisson A.W., "Input impedance of dielectric resonator antennas excited by coaxial probe," IEEE Trans. Antennas Propagation, 1994, 42, 960-966.

[5] Leung K.W., and Ng H.K., "The slot-coupled hemispherical dielectric resonator antenna with a parasitic patch: Applications to the circularly polarized antenna and wideband antenna," IEEE Trans. Antennas Propagation, 2005, 53(5), 1762-1769.

[6] Al Salameh M.S., Antar Y.M.M., and Seguin G.,"Coplanar-waveguide-fed slot-coupled rectangular dielectric resonator antenna," IEEE Trans. Antennas Propagation, 2002, 50(10), 1415-1419.

[7] Kishk A.A., Ahn B., and Kajfez D., "Broadband stacked dielectric resonator antennas," IEEE Electron. Lett., 1989, 25(18), 12311233.

[8] Petosa A., Simons N., Siushansian R., Ittipiboon A., and Cuhaci,M., "Design and analysis of multisegment dielectric resonator antennas," IEEE Trans. Antennas Propagation, 2000, 48(5), 738-742. 